\title{
Message from the Editor
}

\author{
J. Bogousslavsky, Lausanne
}

It is the policy of the journal to rotate the members of the Editorial Board every now and then and I would like to thank the following people for their great efforts over the past years:

C. Bassetti, Zürich

D. Dressler, Rostock

J. Ghika, Lausanne

J. Sander, Gerrards Cross

P. Scheltens, Amsterdam

A. Yamadori, Sendai

I would also like to welcome the new members of the Editorial Board and thank them for agreeing to support European Neurology:

D. Adams, Paris

E.B. Bromfield, Boston, Mass.

P. Giannakopoulos, Genève

O. Godefroy, Amiens

K. Hess, Zürich

R.N. Kalaria, Newcastle upon Tyne

S. Oppenheimer, Washington, D.C.

The following names are those of ad hoc reviewers, to whom I would also like to express my gratitude (January 2003 - October 2003):

\section{J.A. Aarli, Bergen, Norway}

H. Adams, Iowa City, Iowa, USA

J.-M. Annoni, Genève, Switzerland

Z. Argov, Jerusalem, Israel

J.-C. Baron, Cambridge, UK

A. Barth, Bern, Switzerland

C. Bassetti, Zürich, Switzerland
R.W. Baumgartner, Zürich, Switzerland

S.R. Benbadis, Tampa, Fla., USA

R. Benecke, Rostock, Germany

T.D. Bird, Seattle, Wash., USA

W.T. Blume, London, Ont., Canada

F.-X. Borruat, Lausanne, Switzerland

M.-G. Bousser, Paris, France

A. Brice, Paris, France

A. Bronstein, London, UK

E. Broussolle, Lyon, France

L. Bruggimann, Lausanne, Switzerland

J.M. Burgunder, Singapore, Singapore

L.R. Caplan, Boston, Mass., USA

A. Carolei, L'Aquila-Coppito, Italy

A. Carota, Genève, Switzerland

E. Carrera, Lausanne, Switzerland

H. Chabriat, Paris, France

M. Clanet, Toulouse, France

S. Clarke, Lausanne, Switzerland

G. Comi, Milano, Italy

C. Confavreux, Lyon, France

A. Culebras, Syracuse, N.Y., USA

S. Di Donato, Milano, Italy

S. Dieguez, Lausanne, Switzerland

H.-C. Diener, Essen, Germany

P.Y. Dietrich, Genève, Switzerland

K. Diserens, La Sarraz, Switzerland

D.W. Droste, Luxembourg, Luxembourg

G.C. Ebers, Oxford, UK

A. Engel, Rochester, Minn., USA

M. Ferrari, Leyden, The Netherlands

J.M. Ferro, Lisboa, Portugal

A. Filla, Napoli, Italy

M. Fisher, Worcester, Mass., USA

\section{KARGER}

(C) 2004 S. Karger AG, Basel

Fax + 41613061234

E-Mail karger@karger.ch

www.karger.com
Accessible online at: www. karger.com/ene 
J. Ghika, Lausanne, Switzerland

P. Giannakopoulos, Genève, Switzerland

M. Glatzel, Zürich, Switzerland

P.J. Goadsby, London, UK

O. Godefroy, Amiens, France

D.I. Graham, Glasgow, UK

F. Graus, Barcelona, Spain

V. Hachinski, London, Ont., Canada

R.G. Hart, San Antonio, Tex., USA

R. Häusler, Bern, Switzerland

H. Henon, Lille, France

K. Hess, Zürich, Switzerland

S. Hesse, Berlin, Germany

J. Hildebrand, Bruxelles, Belgium

L. Hirt, Lausanne, Switzerland

C. Hock, Zürich, Switzerland

D. Inzitari, Firenze, Italy

P. Jichlinski, Lausanne, Switzerland

S. Joray, Lausanne, Switzerland

T. Karapanayiotides, Thessaloniki, Greece

J.S. Kim, Seoul, South Korea

J. Kimura, Kyoto, Japan

A.D. Korczyn, Ramat Aviv, Israel

T. Kuntzer, Lausanne, Switzerland

T. Landis, Genève, Switzerland

B. Laurent, Saint-Etienne, France

K.L. Leenders, Groningen, The Netherlands

N. Leigh, London, UK

G.L. Lenzi, Roma, Italy

D. Leys, Lille, France

K.-O. Lovblad, Genève, Switzerland

P.A. Lyrer, Basel, Switzerland

P. Maeder, Lausanne, Switzerland

M.-H. Mahagne, Nice, France

J.C. Masdeu, Pamplona, Spain

J. Mayer, Lausanne, Switzerland

O. Meienberg, Binningen, Switzerland

C. Michel, Genève, Switzerland

P. Michel, Lausanne, Switzerland

H.W. Moser, Baltimore, Md., USA

R. Müri, Bern, Switzerland

B. Nater, Lausanne, Switzerland

W.H. Oertel, Marburg, Germany

S.M. Oppenheimer, Washington, D.C., USA
F. Perren, Mannheim, Germany

J. Philippe, Genève, Switzerland

C. Pierrot-Deseilligny, Paris, France

B. Pieschowski, Lausanne, Switzerland

W. Poewe, Innsbruck, Austria

E.-W. Radü, Basel, Switzerland

M. Reichhart, Lausanne, Switzerland

H. Reichmann, Dresden, Germany

J. de Reuck, Gent, Belgium

A. Rossetti, Lausanne, Switzerland

J. Röther, Hamburg, Germany

L. Rumbach, Besançon, France

H. Russmann, Lausanne, Switzerland

M.A. Samuels, Boston, Mass., USA

J.W.A.S. Sander, Gerrards Cross, UK

P. Scheltens, Amsterdam, The Netherlands

M. Schluep, Lausanne, Switzerland

J. Schoenen, Liège, Belgium

G. Schroth, Bern, Switzerland

S. Schwab, Heidelberg, Germany

Y. Shinohara, Kanagawa, Japan

C.J.M. Sindic, Bruxelles, Belgium

F. Spertini, Lausanne, Switzerland

A.J. Steck, Basel, Switzerland

R. Stupp, Lausanne, Switzerland

M. Sturzenegger, Bern, Switzerland

F. Taroni, Milano, Italy

B.E. Tettenborn, St. Gallen, Switzerland

J. Theron, Caen, France

P. Tonali, Roma, Italy

N. de Tribolet, Lausanne, Switzerland

L. Urben, Lausanne, Switzerland

J.M. Vallat, Limoges, France

M. Vidailhet, Paris, France

A. Vincent, Oxford, UK

P. Vuadens, Sion, Switzerland

P. Vuilleumier, Genève, Switzerland

J.H.J. Wokke, Utrecht, The Netherlands

A. Yamadori, Sendai, Japan

Y. Yonekawa, Zürich, Switzerland 